\title{
Increased Th17 cells and IL-17 in rats with traumatic optic neuropathy
}

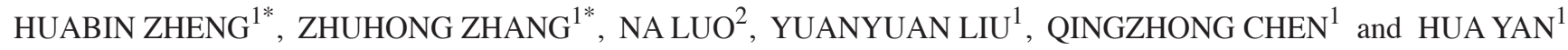 \\ ${ }^{1}$ Department of Ophthalmology, Tianjin Medical University General Hospital, Tianjin 300052; \\ ${ }^{2}$ Department of Immunology, School of Medicine, Nankai University, Tianjin 300071, P.R. China
}

Received September 24, 2013; Accepted March 17, 2014

DOI: $10.3892 / \mathrm{mmr} .2014 .2448$

\begin{abstract}
T helper 17 (Th17) cells are strong inducers of numerous autoimmune diseases and inflammation. However, the role of Th17 cells and interleukin (IL)-17 in traumatic optic neuropathy (TON) are yet to be elucidated. In the present study, a rat model of TON was established using a fluid percussion brain injury device. Th17 cells were found to be upregulated in the spleens of rats in the TON group. In addition, the level of IL-17 in the retina of rats in the TON group was observed to increase with the upregulation of the Th17 cells. Furthermore, the expression of IL-17 in the optic nerve was found to be upregulated between one and seven days following injury in the rats in the TON group. These findings strongly suggest that the ratio of Th17 cells and the expression of IL-17 are upregulated in rats with TON. These findings also provide a rationale for developing therapeutic agents to treat TON.
\end{abstract}

\section{Introduction}

Traumatic optic neuropathy (TON) is a serious injury of the optic nerve, which involves the disruption of visual function. The injury associated with TON may be permanent or temporary. Etiological factors associated with TON include head trauma due to motor vehicle and bicycle accidents, falls or assault. TON is a type of head trauma (1) and may be divided into direct and indirect injuries. Direct optic nerve injury is less common, due to the protection provided by the bony orbit (2). At present, the pathogenesis, treatment and prognosis of TON remain to be elucidated. It has been reported that injury may activate the immune system, which may influence the prognosis of the disease (3).

Correspondence to: Professor Hua Yan, Department of Ophthalmology, Tianjin Medical University General Hospital, 154 Anshan Road, Tianjin 300052, P.R. China

E-mail: phuayan2000@163.com

${ }^{*}$ Contributed equally

Key words: traumatic optic neuropathy, $\mathrm{T}$ helper 17 cells, interleukin-17, retina, optic nerve
Cluster of differentiation (CD) $4^{+} \mathrm{T}$ helper (Th) cells are divided into pro- and anti-inflammatory Th1 and Th2 cells, respectively, based on their secretory cytokines (4). Interleukin (IL)- 6 and transforming growth factor (TGF)- $\beta$ induce naïve $\mathrm{CD}^{+}{ }^{+} \mathrm{T}$ cells into Th17 cells. Th17 cells secrete IL-1 (5) and are involved in the pathology of various autoimmune diseases, including neuromyelitis optica (6), rheumatoid arthritis and multiple sclerosis (7). High concentrations of TGF- $\beta$ induce the development of regulatory T cells (Tregs) and alterations in the Th17/Treg ratio have been reported in several autoimmune diseases. Th17 cells are involved in immunological responses and induce inflammation (8). IL-17 primarily functions to activate macrophages and promote the secretion of inflammatory cytokines, including TNF- $\alpha$ and IL-1 $\beta$ (9).

In the present study, an animal model of TON was established in rats using a fluid percussion brain injury (FPI) device and the rate of Th17 cells in the spleen was detected. The expression of IL-17 in the optic nerve and the levels of IL-17 in the retina were also detected. It was hypothesized that IL-17 may regulate inflammation in TON.

\section{Materials and methods}

Chemicals. Lymphocyte separation medium was purchased from Haoyang Biological Manufacture Co., Ltd (Tianjin, China). Dulbecco's Modified Eagle Medium (DMEM) and fetal bovine serum were purchased from Invitrogen Life Technologies, (Carlsbad, CA, USA). CD4 and IL-17 monoclonal antibodies were obtained from BD Biosciences (San Diego, CA USA).

Induction of TON and treatment protocols. Sixty Sprague-Dawley rats (age, 4 weeks) were purchased from the Military Medical Academy of China (Beijing, China). Rats were randomly divided into normal, control and TON groups. The normal and control groups consisted of 10 rats and the experimental TON group consisted of 40 rats. The TON group was divided into four subgroups (1,7, 14 and 28 days) of 10 rats on the basis of different observation times. Rats in the normal group received no treatment and those in the control group received the same treatment as the TON groups, but without the induction of TON. In the rats in the experimental TON groups, the right eyes were considered experimental eyes and the left eyes received no treatment. The TON model was established 
Table I. Changes in latent period and amplitude in flash-visual evoked potential examination.

\begin{tabular}{lccc}
\hline Group & Latent period $(\mathrm{ms})$ & P-value $^{\mathrm{a}}$ & Amplitude $(\mu \mathrm{V})^{\mathrm{P}-\text { value }^{\mathrm{b}}}$ \\
\hline Normal & $78.80 \pm 8.52$ & & $12.23 \pm 1.74$ \\
Control & $79.70 \pm 9.79$ & & $13.45 \pm 2.21$ \\
TON 1 day & $93.60 \pm 12.50$ & $<0.001$ & $10.41 \pm 1.75$ \\
TON 7 days & $112.00 \pm 9.46$ & $<0.001$ & $7.50 \pm 1.34$ \\
TON 14 days & $126.30 \pm 10.48$ & $<0.001$ & $5.89 \pm 1.29$ \\
TON 28 days & $129.30 \pm 8.23$ & $<0.001$ & $5.61 \pm 1.09$ \\
\hline
\end{tabular}

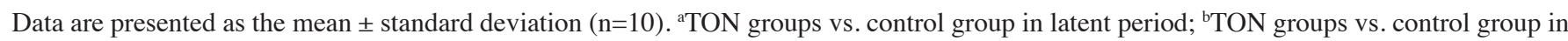
amplitude. TON, traumatic optic neuropathy.

in accordance with the method described previously (10). In brief, rats were anaesthetized using $10 \%$ chloral hydrate. The conjunctivas were cut and FPI was used to induce trauma. A tube of optic nerve was then inserted through the conjunctival incision and placed $\sim 2 \mathrm{~mm}$ into the conjunctival cleft. The hammer angle was $25^{\circ}$. Subsequent to injury, the right eyes were treated with Levofloxacin (Santen Pharmaceutical Co., Osaka, Janpan) each day.

Flash-visual evoked potential (F-VEP) examination. F-VEP was performed on the eyes. Rats were anesthetized using $10 \%$ chloral hydrate prior to examination. A RETI visual electrophysiology system (Roland Consult, Brandenburg an der Havel, Germany) with silver needle electrodes was used. The recording and reference electrodes were inserted subcutaneously into the rat occipital tuberosity (OZ position). The ground electrode was inserted subcutaneously into the ear mastoid. Full visual field white flash stimulation was applied, at a stimulation frequency of $1.6 \mathrm{~Hz}$ and a band pass width of $30-100 \mathrm{~Hz}$, for $250 \mathrm{~ms}$ per rat. The waveform was superimposed 100 times. Each rat was recorded three times with an interval of $10 \mathrm{~min}$. The parameters recorded were F-VEP latent period LP2 (the P2 wave response time, ms) and amplitude $\mathrm{N} 2-\mathrm{P} 2(\Delta$ between $\mathrm{N} 2$ wave trough and $\mathrm{P} 2$ wave peak, $\mu \mathrm{V}$ ). Three measurements were averaged for each parameter.

Flow cytometry. A splenocyte suspension was generated from rat spleen samples and the lymphocytes were isolated using lymphocyte separation medium. Lymphocytes were collected and washed twice with DMEM and transferred onto 24-well plates at a concentration of $1 \times 10^{7}$ cells $/ \mathrm{ml}$. A total of $50 \mathrm{ng} / \mathrm{ml}$ phorbol ester and $1 \mu \mathrm{g} / \mathrm{ml}$ ionomycin were added to the suspension. Following $5 \mathrm{~h}$ of culture at $37^{\circ} \mathrm{C}$, lymphocytes were collected. Cells were incubated using fluorescein isothiocyanate (FITC)-conjugated monoclonal antibodies against rat $\mathrm{CD}^{+}$and R-phycoerythrin-conjugated monoclonal antibodies against rat IL-17 for $30 \mathrm{~min}$ at room temperature. Impurities were then filtered from the cells and the number of Th17 cells in spleen was detected using a flow cytometer (BD Bioscieces, San Diego, CA, USA).

Immunohistochemistry. IL-17 staining was performed on rat retinas using a monoclonal antibody against IL-17 at a dilution of 1:100 and an Immunocruz ${ }^{\circledR}$ staining system (Santa Cruz
Biotechnology, Inc., CA, USA) according to the manufacturer's instructions.

Western blot analysis. Optic nerve and tissue lysates were homogenized and proteins were extracted from the optic nerve according to the manufacturer's instructions (Sigma-Aldrich, Munich, German). A total of $20 \mu \mathrm{g}$ protein was electrophoresed using $12 \%$ SDS-PAGE and transferred to polyvinylidene fluoride (PVDF) membranes. Following blocking with $25 \mathrm{ml}$ $5 \%$ non-fat milk and washing with Tris-buffered saline with Tween-20 solution three times, PVDF membranes were probed overnight at $4^{\circ} \mathrm{C}$ with anti-IL-17 and anti- $\beta$-actin antibodies, diluted 1:500 and 1:1,000, respectively. Subsequent to washing, membranes were incubated with horseradish peroxidase-conjugated anti-rabbit immunoglobulin G secondary antibodies diluted 1:5,000 for $1 \mathrm{~h}$ at room temperature. Proteins were then detected using an enhanced chemiluminescence system (Millipore, Billerica, MA, USA) and X-ray film (Thermo, Rockford, IL, USA).

Statistical analysis. All statistical analyses were performed using the SPSS 17.0 statistical software package (SPSS, Inc., Chicago, IL, USA). Baseline characteristics for all groups are presented as the mean \pm standard deviation. Differences between groups were analyzed using Student's paired t-test or analysis of variance, as appropriate. $\mathrm{P}<0.05$ was considered to indicate a statistically significant difference.

\section{Results}

F-VEP examination of the TON model. To determine the establishment of the TON rat model, F-VEP examinations were performed. Rats in all groups were observed to have NPN waveforms, which the P2 and N2-P2 waveforms were observed markedly. If the latent period of $\mathrm{P} 2$ was prolonged and the amplitude of N2-P2 was reduced, these means optic nerve was injured. Following optic nerve injury, the latent period was markedly prolonged and the amplitude was significantly reduced until $\sim 14$ days, following which the waveforms stablized (Fig. 1). No significant difference was identified in the latent period between the rats in the normal and control groups $(\mathrm{P}=0.829)$. However, the differences in latent period and amplitude between the rats in the control and experimental TON groups were found to be statistically significant (Table I). 


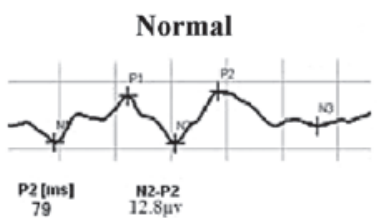

D

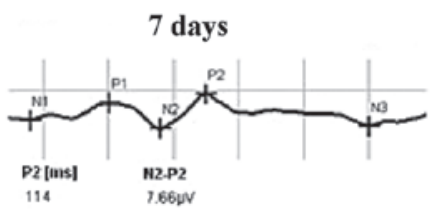

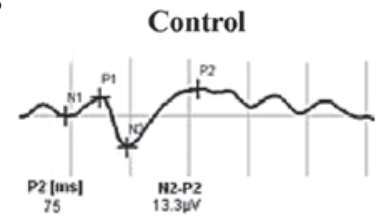

$\mathbf{E}$

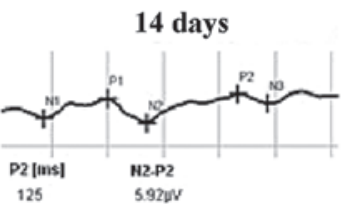

C

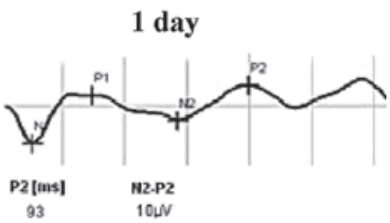

$\mathbf{F}$

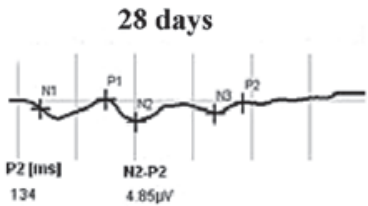

Figure 1. F-VEP examination of the TON model in rats. F-VEP examination in the (A) normal and (B) control groups and the TON groups at (C) one, (D) seven, (E) 14 and (F) 28 days subsequent to the induction of injury. F-VEP, flash-visual evoked potential.

A

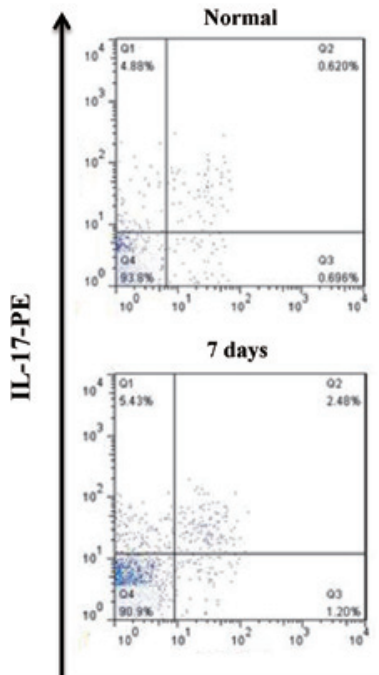

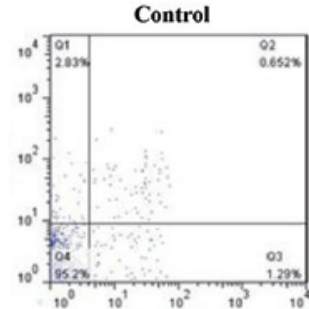

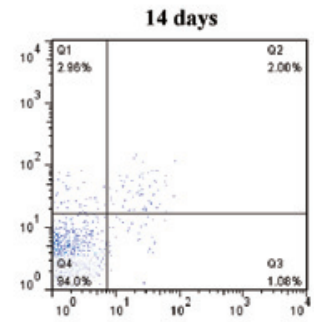

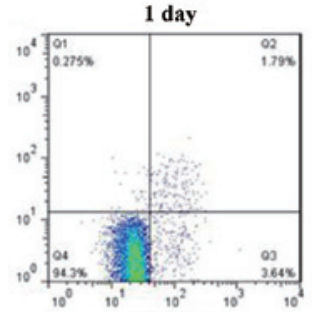

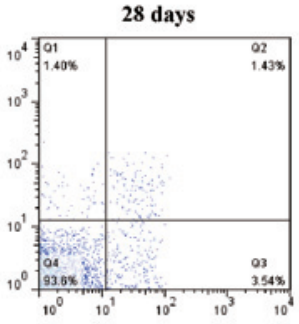

B

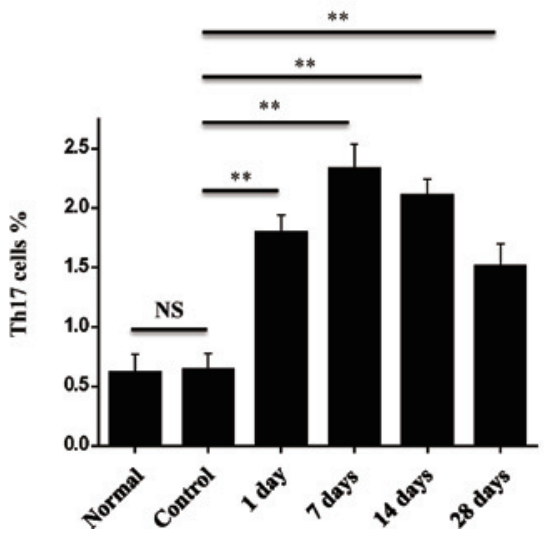

Figure 2. Th17 cell regulation in rats with TON. Splenocytes from rats in the normal, control and TON groups were analyzed for Th17 cells using flow cytometry. (A) Ratio of Th17 cells in the spleen of the rats in the normal, control and TON treatment groups 1, 7, 14 and 28 days subsequent to the induction of injury. (B) Bar graph of the results of flow cytometry. ${ }^{* * *} \mathrm{P}<0.01$ vs. control. TON, traumatic optic neuropathy; Th17, T helper 17; IL, interleukin.

These findings suggest that the TON model was established successfully in the rats in the experimental groups.

Changes in Th17 cells in the spleens of rats with TON. In order to investigate the changes in the Th17 cells in the spleens of the rats with TON, flow cytometry was performed. Th17 cells were identified using FITC-conjugated monoclonal antibodies against rat $\mathrm{CD}^{+}$and $\mathrm{PE}$-conjugated monoclonal antibodies against rat IL-17. As shown in Fig. 2A, the ratio of Th17 cells in the spleens of the rats in the control group was $0.94 \pm 0.13 \%$, which increased one day following injury and peaked seven days following injury (Fig. 2A and B). The ratio of Th17 cells in 
A
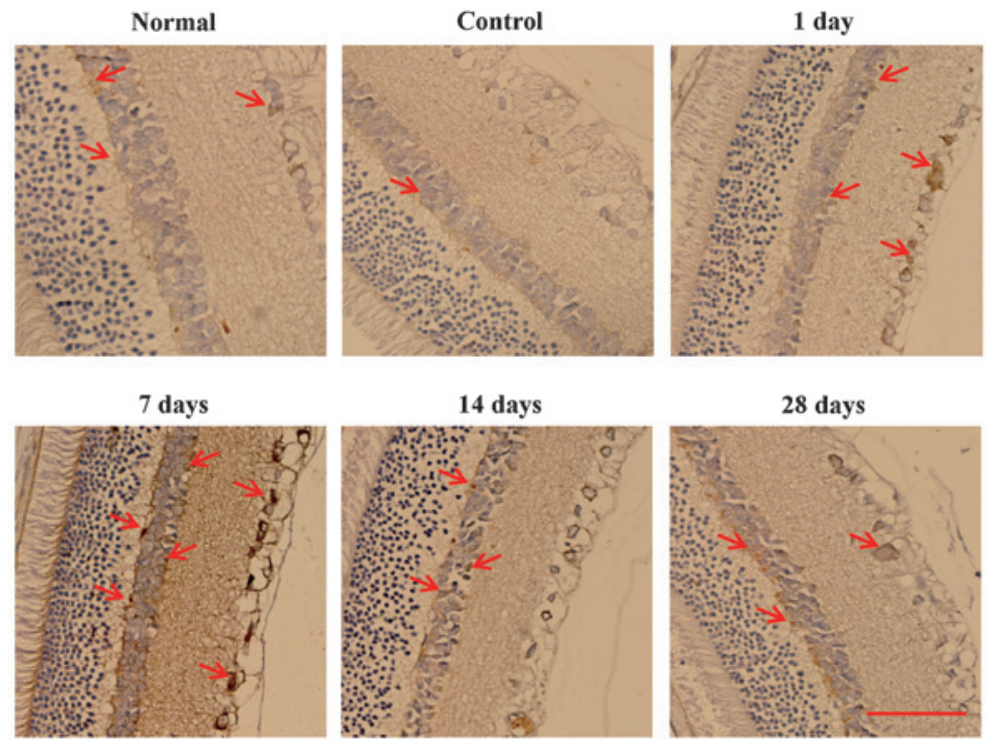

$\mathbf{B}$

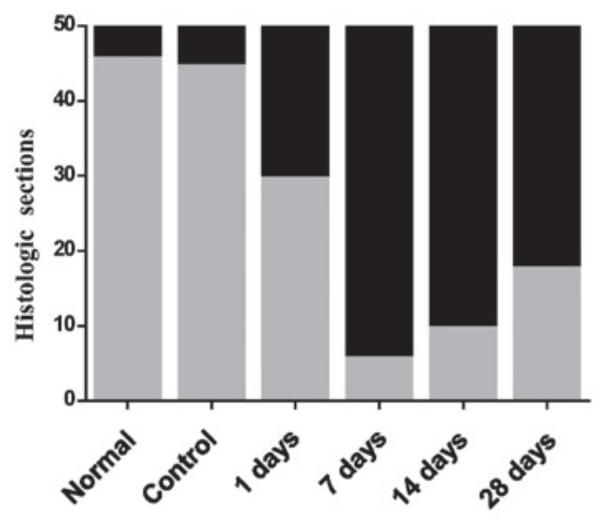

$\mathbf{C}$

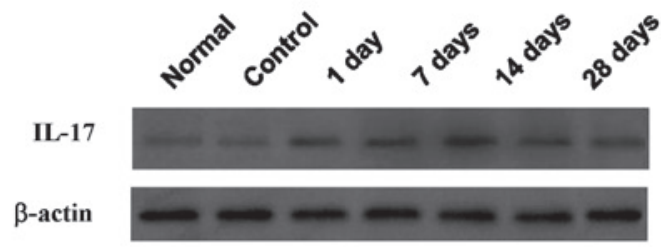

Figure 3. Regulation of IL-17 in rats with TON. (A) Immunohistochemistry of IL-17 levels in the retina of the rats with TON. (B) Bar graph of the results of immunohistochemistry. (C) Western blot analysis of IL-17 levels in the optic nerve of the rats with TON. Scale bar, $100 \mu \mathrm{m}$. IL, interleukin; TON, traumatic optic neuropathy.

the spleens of the TON rats was observed to decrease at 14 and 28 days following injury, but remained higher than that in the rats in the control group (Fig. 2). These findings suggest that the ratio of Th17 cells in the spleen was increased in the TON rats.

Changes in IL-17 in the retina and optic nerve of rats with TON. Th17 cells secrete IL-17, which regulates inflammation. Therefore, the levels of IL-17 in the retina were analyzed. Immunohistochemistry revealed that the levels of IL-17 increased in the retina of the TON rats and peaked seven days following the induction of injury. Results were classified as either moderate ( $0 \%$ to $<25 \%$, gray area) or strong $(>25 \%$, dark area) (Fig. 3B). Compared with the seven day group, levels of IL-17 were observed to be decreased in the TON rats in the 14 and 28 day groups (Fig. 3A and B), but remained higher than that in the rats in the control group.
In order to further investigate the changes in IL-17 in the optic nerve, the expression of IL-17 was assessed using western blot analysis. IL-17 was observed to be expressed in the rats in all groups (Fig. 3C), but was lower in the rats in the normal and control groups than those in the TON groups. Following the induction of injury, the expression of IL-17 was observed to increase initially, peak at seven days then decrease. The differences in IL-17 expression between the control and the TON groups was statistically significant. These findings show that the pattern of IL-17 expression correlates with the trend in the ratio of Th17 cells.

\section{Discussion}

TON often occurs in patients with closed head injury. Damage to the optic nerve includes immediate and secondary damage in a 
proportion of retinal ganglion cells (11). It is difficult to treat the immediate damage to the optic nerve (12) and it is important to suppress secondary damage (13). A previous study reported that patients with TON were treated with high doses of intravenous steroids (14). In the present study, the immunologic mechanism underlying TON was investigated in order to identify a novel, efficient method for the therapeutic treatment of TON.

In the present study, FPI was used to establish an animal model of TON, which has advantages with regard to experimental repeatability and stability (15). Following the generation of the model, F-VEP revealed that the latent period was increased and the amplitude was significantly reduced until 14 days following the induction of injury. These findings show that the animal model was successfully established.

The pathogenesis of TON is complex. Clinical and experimental studies have revealed that optic nerve damage involves numerous mechanisms, which have yet to be elucidated $(12,16)$. There is no proven effective treatment for TON. High dose corticosteroids and decompressing surgery have been adopted in clinical treatment $(1,17)$. Therefore, it is important to investigate the pathogenesis of TON in order to identify an effective treatment. The immunologic response has been suggested to be involved in the pathogenesis of TON. Kipnis et al (18) observed certain beneficial or destructive autoimmunity following injury. Furthermore, immunization with myelin basic proteins emulsified in complete or incomplete Freund's adjuvant was found to protect optic nerve neurons from secondary degeneration following crush injury. Fisher et al (19) reported that IL-6 inhibited neuronal cell survival. Whether immune-derived factors are beneficial or harmful to nerve recovery depends on the phenotype of the immune cells and the timing of the interaction with the damaged neural tissue. Based on these reports, in the present study, it was hypothesized that the ratio of Th17 cells increases in the TON model.

In the present study, dynamic changes in the level of IL-17 were observed in the retina and optic nerves of TON rats, which correlated with the trend in the ratio of Th17 cells in the spleen. The levels of Th17 cells and IL-17 initially increased upon induction of TON, peaking at 7 days and decreasing at 14 and 28 days subsequent to the induction of injury. Th17 cells are highly auto-pathogenic and induce tissue inflammation and autoimmune diseases (20). Th17 cells secrete IL-17, -22 and -21 and express the IL-23 receptor (21). IL-17 is a cytokine with numerous pro-inflammatory functions and is likely to be involved in either the causation or progression of inflammatory diseases and transplant rejection in humans (22). Peng et al (23) identified that it is possible to detect Th17 cells among activated auto-reactive and bystander T cells and that Th17 cells may have a key role in the pathogenesis of experimental autoimmune uveoretinitis. The results of the present study are consistent with those of previous reports, which suggest that Th17 cells and IL-17 may induce or promote inflammation in TON.

In conclusion, the present study has shown the changes in Th17 cells and IL-17 in TON rats. Furthermore, the findings suggest that the ratio of Th17 cells in the spleen and the expression of IL-17 in the retina and optic nerve increased in rats with TON. These findings demonstrate that Th17 cells may participate in and promote the progression of TON.

\section{Acknowledgements}

This study was supported by the Tianjin application infrastructure and cutting-edge technology research program of China (grant no. 10JCZDJC20300)

\section{References}

1. Yu Wai Man P and Griffiths PG: Surgery for traumatic optic neuropathy. Cochrane Database Syst Rev 6: CD005024, 2013.

2. Miliaras G, Fotakopoulos G, Asproudis I, Voulgaris S, Zikou A and Polyzoidis K: Indirect traumatic optic neuropathy following head injury: report of five patients and review of the literature. J Neurol Surg A Cent Eur Neurosurg 74: 168-174, 2013.

3. Schwartz M: Optic nerve crush: protection and regeneration. Brain Res Bull 62: 467-471, 2004.

4. Mosmann TR, Cherwinski H, Bond MW, Giedlin MA and Coffman RL: Two types of murine helper T cell clone. I. Definition according to profiles of lymphokine activities and secreted proteins. 1986. J Immunol 175: 5-14, 2005.

5. Bettelli E, Carrier Y, Gao W, et al: Reciprocal developmental pathways for the generation of pathogenic effector TH17 and regulatory T cells. Nature 441: 235-238, 2006.

6. Li Y, Wang H, Long Y, Lu Z and Hu X: Increased memory Th17 cells in patients with neuromyelitis optica and multiple sclerosis. J Neuroimmunol 234: 155-160, 2011.

7. Ortega C, Fernández S, Estévez OA, Aguado R, Molina IJ and Santamaria M: IL-17 producing T Cells in celiac disease: angels or devils? Int Rev Immunol 32: 534-543, 2013.

8. Miossec P, Korn T and Kuchroo VK: Interleukin-17 and type 17 helper T cells. N Engl J Med 361: 888-898, 2009.

9. Dragon S, Saffar AS, Shan L and Gounni AS: IL-17 attenuates the anti-apoptotic effects of GM-CSF in human neutrophils. Mol Immunol 45: 160-168, 2008.

10. Yan H, Li F and Zhang L: A new and reliable animal model for optic nerve injury. Curr Eye Res 37: 941-948, 2012.

11. Negi A: New insights into the study of optic nerve diseases. Nihon Ganka Gakkai Zasshi 117: 187-210, 2013 (In Japanese).

12. Ropposch T, Steger B, Meço C, et al: The effect of steroids in combination with optic nerve decompression surgery in traumatic optic neuropathy. Laryngoscope 123: 1082-1086, 2013.

13. Shi W, Wang HZ, Song WX, Yang WL, Li WY and Wang NL: Axonal loss and blood flow disturbances in the natural course of indirect traumatic optic neuropathy. Chin Med J (Engl) 126: 1292-1297, 2013.

14. Yu Wai Man P and Griffiths PG: Steroids for traumatic optic neuropathy. Cochrane Database Syst Rev 6: CD006032, 2013.

15. Thompson HJ, Lifshitz J, Marklund N, et al: Lateral fluid percussion brain injury: a 15-year review and evaluation. J Neurotrauma 22: 42-75, 2005.

16. Bodanapally UK, Kathirkamanathan S, Geraymovych E, et al: Diagnosis of traumatic optic neuropathy: application of diffusion tensor magnetic resonance imaging. J Neuroophthalmol 33: 128-133, 2013.

17. Steinsapir KD and Goldberg RA: Traumatic optic neuropathy: an evolving understanding. Am J Ophthalmol 151: 928-933, 2011.

18. Kipnis J, Mizrahi T, Yoles E, Ben-Nun A and Schwartz M: Myelin specific Th1 cells are necessary for post-traumatic protective autoimmunity. J Neuroimmunol 130: 78-85, 2002.

19. Fisher J, Mizrahi T, Schori H, et al: Increased post-traumatic survival of neurons in IL-6-knockout mice on a background of EAE susceptibility. J Neuroimmunol 119: 1-9, 2001.

20. Basu R, Hatton RD and Weaver CT: The Th17 family: flexibility follows function. Immunol Rev 252: 89-103, 2013.

21. Dardalhon V, Korn T, Kuchroo VK and Anderson AC: Role of Th1 and Th17 cells in organ-specific autoimmunity. J Autoimmun 31: 252-256, 2008

22. Afzali B, Lombardi G, Lechler RI and Lord GM: The role of T helper 17 (Th17) and regulatory T cells (Treg) in human organ transplantation and autoimmune disease. Clin Exp Immunol 148: 32-46, 2007.

23. Peng Y, Han G, Shao H, Wang Y, Kaplan HJ and Sun D: Characterization of $\mathrm{IL}-17^{+}$interphotoreceptor retinoid-binding protein-specific $\mathrm{T}$ cells in experimental autoimmune uveitis. Invest Ophthalmol Vis Sci 48: 4153-4161, 2007. 